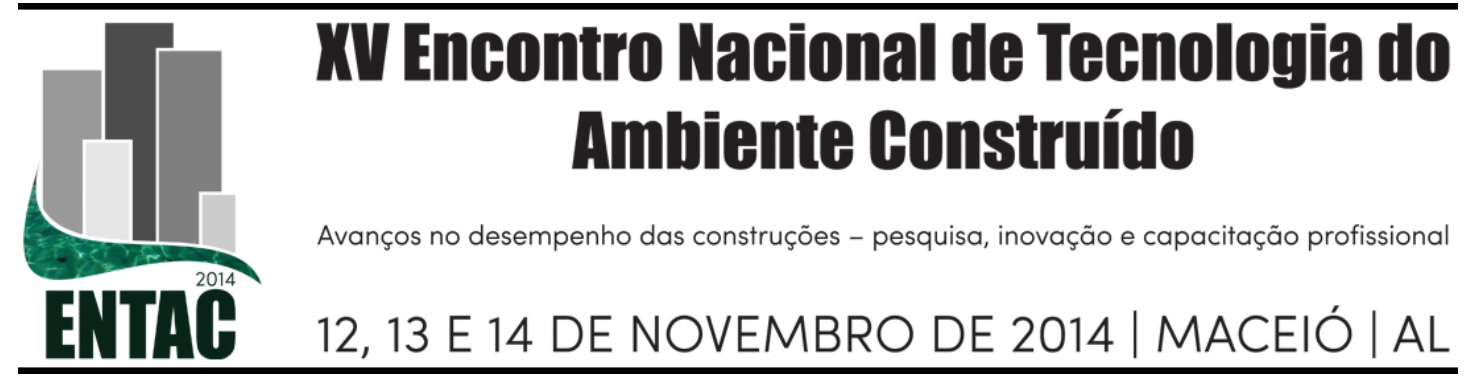

\title{
GERAÇÃO DE MAPAS DE DANOS DE FACHADAS A PARTIR DE PROCESSAMENTO DIGITAL DE IMAGENS
}

\author{
MELO JR., Carlos Mariano (1); ALVES, Gelson de Sousa (2); EVANGELISTA \\ JR, Francisco (3); CHRISTAKOU, Evangelos Dimitros (4); SILVA, Lenildo \\ Santos da (5); NEPOMUCENO, Antônio Alberto (6)
}

(1) Universidade de Brasília, e-mail: carlosmmjunior@gmail.com (2) Universidade de Brasília, e-mail: gelsondsalves@gmail.com (3) Universidade de Brasília, e-mail: fejr@unb.br (4) Universidade de Brasília, e-mail: vangelis@unb.br (5) Universidade de Brasília, e-mail: lenildo_s@ hotmail.com

(6) Universidade de Brasília, e-mail: aa.nepomuceno@gmail.com

\begin{abstract}
RESUMO
Os mapas de danos de fachadas são representações gráficas que objetivam identificar e discriminar as manifestações patológicas, sintetizando as alterações de condições operacionais e estruturais de partes desse sistema. Atualmente, as imagens fotográficas digitais não se limitam à documentação do estado dos edifícios, pois a elevada qualidade das câmeras fotográficas digitais e a existência de ferramentas numéricas possibilitam o desenvolvimento de novos métodos, como a aplicação de processamento de imagem. O processamento digital de imagens é o conjunto de técnicas para a manipulação de imagens, objetivando facilitar a extração de informações. Diante da contextualização, o presente trabalho visa mapear e quantificar manchas escuras em fachadas por meio de processamento digital de imagens em uma ferramenta numérica implementada no MATLAB. Os dados apresentados são baseados em resultados parciais. Para realização da pesquisa experimental, foi utilizada para a aquisição das imagens das fachadas da Biblioteca Central da UnB uma câmera fotográfica digital de 20,2 megapixels acoplada em veículo aéreo não tripulado (VANT). Posteriormente à aquisição das imagens, essas foram processadas no programa de fotogrametria digital para geração de ortoimagens, as quais foram carregadas no MATLAB. O mapeamento de manchas foi realizado por meio do código para detecção do contorno, padrões de cores e valor de área mínima desejada. Os mapas de danos das fachadas foram criados com sucesso e fazendo também uso de informações sobre o percentual quantitativo de manchas escuras em diferentes intervalos de área em escalas de $\mathrm{cm}^{2}$.
\end{abstract}

Palavras-chave: Fachadas, Mapa de danos, Processamento digital de imagens.

\begin{abstract}
Damage maps are graphical representations that aim to identify and classify pathological manifestations in building facades in order to classify the operational and structural changes of the system condition. Currently, digital photography images are not limited to only register the state of the buildings since the high quality digital cameras and the existence of numerical tools enable the development of new methods of image processing. The digital image processing is the set of techniques for manipulating images, aiming to facilitate the extraction of information. Given the context, this paper aims to map and quantify stains and change of patterns on facades through digital image processing in a numerical tool implemented in MATLAB. The data presented are based on partial results that acquired images of the facades of the Central Library at University of Brasilia using a digital camera of 20.1 megapixels coupled to unmanned aerial vehicle (UAV). After the image acquisition, those were processed to generate orthoimages. The mapping of stains in MATLAB was performed to detect contour, color standards and minimum value desired area. Defects maps of the facades were also created with the quantitative
\end{abstract}


information of the percentage of stain. The results showed the successful quantitative identification and characterization of stains patterns in different ranges of area even in a $\mathrm{cm}^{2}$ scale.

Keywords: Facades, Defects maps, Digital images processing.

\section{INTRODUÇÃO}

A preocupação com o sistema fachada é relevante, pois a integridade diz respeito à permanência do edifício que, para ser vivido, necessita cumprir o tempo previsto para a sua duração sem perdas funcionais ou estéticas (CARRIÓ, 1985). O estudo sistêmico dos danos em fachadas baseado em mapas de identificação de problemas, e consequentemente na suposição das causas e compreensão dos mecanismos, favorece a elaboração de instrumentos preditivos ou corretivos, ou seja, em ações de manutenção ou recuperação do invólucro do edifício.

Nesse propósito, pesquisas têm-se dedicado a compreender os fatores que corroboram o surgimento dos danos em fachadas (PETRUCCI, 2000; FLORES-COLEN, 2009). Alguns desses trabalhos apoiam-se metodologicamente na criação de mapas de danos para fachadas por meio da inspeção visual, seguida da quantificação de manifestações patológicas globais do elemento considerado.

Segundo Bertrand et al. (2003), a inspeção visual é o primeiro passo para a avaliação contínua em edifícios, pois permite que decisões sejam tomadas quanto à necessidade de procedimentos de intervenções em fase de uso. Esse método de verificação caracteriza-se pelo empirismo, pelas experiências adquiridas sobre o comportamento e pela coleta de informações em serviço.

A inspeção visual é etapa preponderante para o mapeamento de danos em fachadas, apoiando-se sobre instrumentos que possam auxiliar no trabalho de campo, como: representação em desenho das fachadas; instrumentos de auxílio para visualização (binóculos, lupas, dentre outros); e equipamentos de registros (máquinas fotográficas). A quantificação dos danos deve ser realizada junto à caracterização e comentários pertinentes aos mesmos, sendo identificados nas representações das fachadas.

Tinoco (2009) comenta que mapa de danos é a representação gráfico-fotográfica, objetivando ilustrar e discriminar, rigorosamente, todas as manifestações de deterioração da edificação, sintetizando, desse modo, o resultado das investigações sobre as alterações de todos os sistemas e componentes do edifício. Barthel et al. (2009) definem o mapa de danos como a representação gráfica do levantamento dos danos identificados no edifício, relacionando-os com os possíveis agentes e causas, citando-se: fissura, degradação por umidade, destacamentos de revestimento, corrosão e outros.

Em pesquisa realizada por Gaspar (2009), a fase de levantamento dos danos envolveu a técnica de desenho à mão para representar as anomalias em fachadas. Por meio de esboços esquemáticos, vantagens adicionais do uso da técnica são apontadas pelo autor, como: maior capacidade do inspetor em memorizar o estudo de caso, facilidade em inserir comentários sobre o edifício e os danos no desenho esquemático e organização prévia, antes do início dos registros fotográficos (Figura 1). 


\section{Figura 1 - Exemplo de desenho esquemático com indicações escritas complementares}

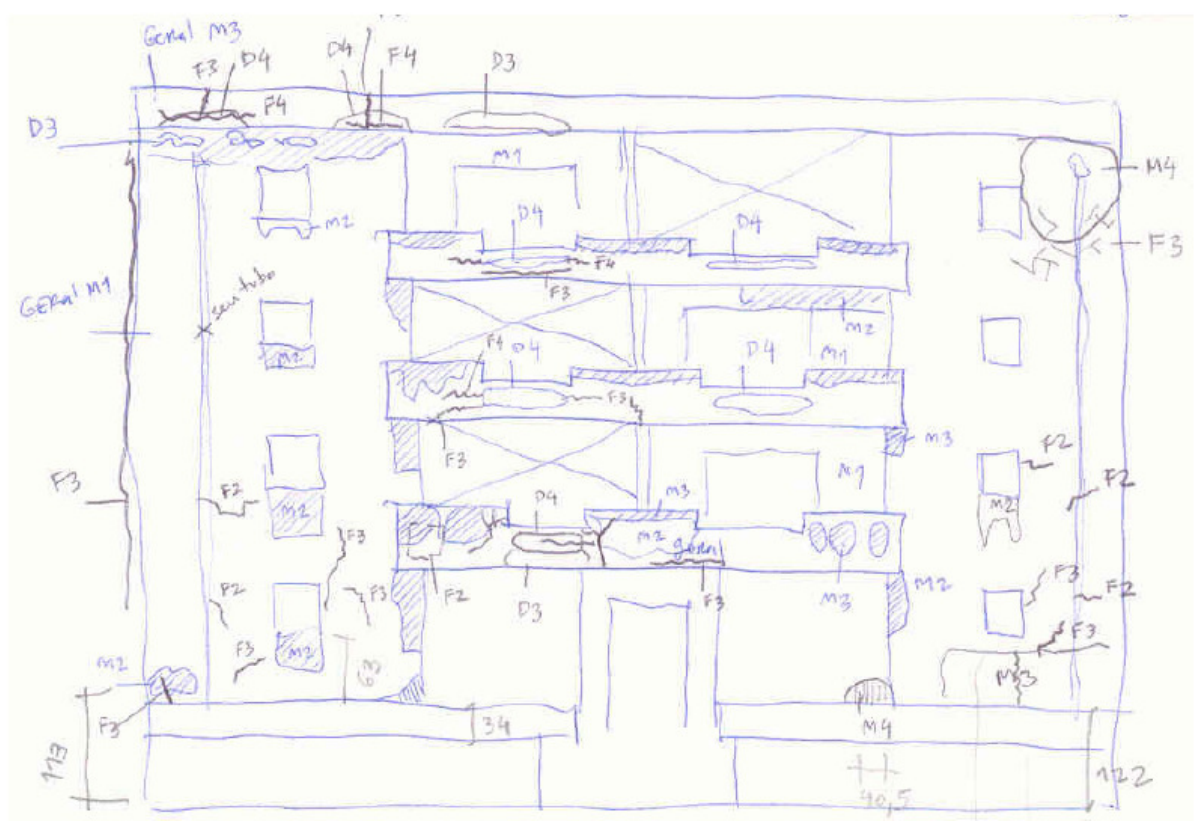

Fonte: Gaspar (2009)

Baseando-se na relevância da elaboração de mapas de danos como instrumento preditivo de ações de manutenção e recuperação de fachadas, a presente pesquisa propõe como objetivo principal o desenvolvimento de uma metodologia de mapeamento e quantificação automática de manchas oriundas de sujidade e crescimento de microrganismos formados sobre superfícies de fachadas com acabamento em concreto aparente por meio do processamento digital de imagens. Dessa maneira, os métodos desenvolvidos visam possibilitar a agilidade na geração de mapas de danos, assim como uma maior precisão dos resultados.

\section{MAPA DE DANOS E PROCESSAMENTO DIGITAL DE IMAGENS}

Costa (2011) enfatiza que o mapa de danos é um material ilustrativo contendo a representação dos componentes construtivos (parede, piso, esquadrias, telhado, etc.) e a deterioração manifestada, assim como as informações necessárias para embasar as atividades seguintes de intervenção e consolidação para os projetos de conservação e restauro. Para tanto, esse tem como forma de apresentação a sobreposição de elementos gráficos, hachuras, fotografias, índices, cores, letras, e legendas contendo dados sobre a incidência dos danos no edifício e suas partes. Nesse sentido, a identificação dos danos, geralmente, é apresentada em representações bidimensionais, tais como plantas ou elevações da edificação, estando os danos ou alterações representados pelas: hachuras; cores; símbolos e números, de forma junta ou separada, representando as informações relacionadas às características dos materiais, dos agentes e causas da degradação.

A tipologia de mapas de danos é descrita como a linguagem gráfica utilizada para representar o dano encontrado, podendo abranger várias formas de representação, tendose as mais usuais: símbolos, linhas, manchas de cores, manchas de texturas, índices de letras ou números, fotografias, mapas com fichas técnicas, ortoimagens ou textos descritivos, além de poder-se associar mais de uma linguagem gráfica num mesmo mapa de dano (COSTA, 2010). 
A forma de representação do dano estará intimamente relacionada com vários aspectos, ou melhor, à maneira de representá-lo no mapa após a inspeção e a detecção pode ser direcionada pelo tipo de manifestação patológica (fissura ou mancha) ou pela forma da representação da edificação (fotografia ou representação gráfica de fachada).

No trabalho desenvolvido por Tinoco e equipe, foi realizado um mapeamento que resultou no mapa de danos em fachada de sobrado tombado pelo IPHAN (Instituto do Patrimônio Histórico e Artístico Nacional) na cidade de Recife, em região remanescente da ocupação urbana no período holandês, no bairro de Santo Antônio. A metodologia utilizada na identificação dos danos foi a inspeção visual e de contato por experiência (FIGURA 2).

\section{Figura 2 - Mapa de identificação de danos}

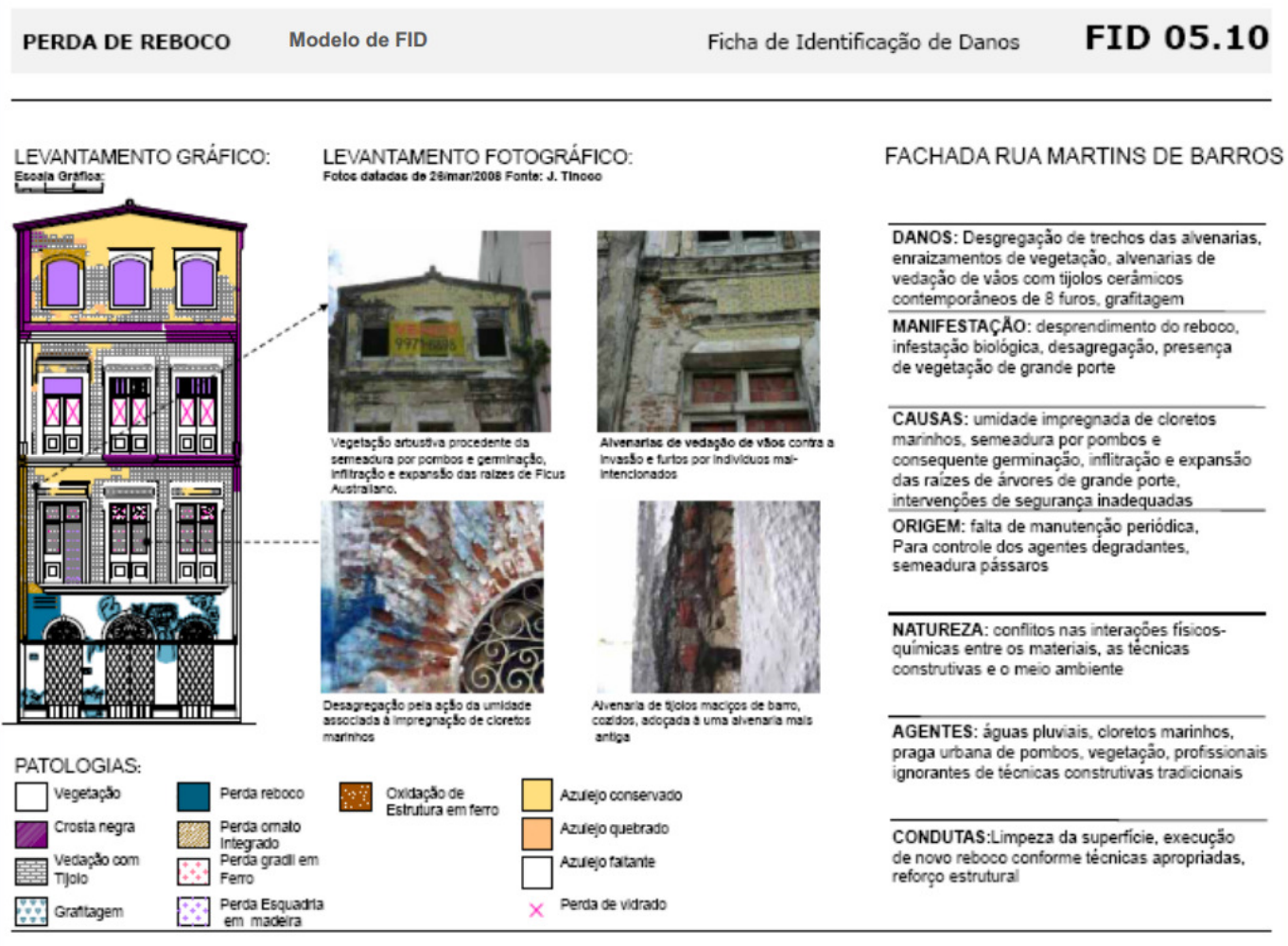

DATA: 26/ MAR/2008 RESP. TECNICO: Jorga Eduardo LUCena TINOCO CREA: 7.128 - DIPE FN COLABORADORES: CeCllia Barthel e Luclana Nepomuceno

Fonte: Tinoco (2009)

Tinoco (2009) ressalva que os mapas de danos devem possuir valor cognitivo, possuindo informações claras e objetivas, não se limitando ao aspecto técnico da comunicação visual e à quantidade de informações. $\mathrm{O}$ mesmo autor discerne que a objetividade e a clareza devem ser alcançadas pela qualidade, contrapondo-se à necessidade de redução e disponibilização das informações indispensáveis à comunicação visual dos danos e de suas respectivas resoluções.

No entanto, os mapas de danos criados pelos autores citados foram elaborados a partir da vetorização da fachada e da representação gráfica das deteriorações em programas CAD (Computer Aided Design), num processo interativo entre software e usuário, tendo-se as imagens fotográficas como ferramenta.

Atualmente, a utilização de imagens fotográficas digitais para a criação de mapas de danos de fachadas é um dos métodos mais utilizados e eficientes como forma de representação. Imagens são definidas como uma função dimensional $f(x, y)$, onde $x$ e $y$ são coordenadas espaciais e a amplitude de $f$ em qualquer par de coordenadas $(x, y)$ é 
chamada de intensidade da imagem nesse ponto. Assim, quando $x, y$ e os valores de $f$ são quantidades discretas e finitas, a imagem é chamada de digital. Uma imagem digital é composta de um número finito de elementos, onde cada elemento possui um valor e uma localização particular, denominado pixel. A imagem truecolor é uma imagem na qual cada pixel é especificado por três valores, sendo um para cada um dos componentes RGB (vermelho, azul e verde) da cor do pixel.

A estrutura básica de informação no MATLAB é a matriz, ou seja, um conjunto ordenado de elementos reais ou complexos. Naturalmente, esse formato é adequado para a representação de imagens, conjuntos ordenados de valor real de cores ou informação de intensidade. A maioria das imagens no MATLAB é armazenada como matrizes bidimensionais, na qual cada elemento de matriz corresponde a um único pixel da imagem exibida.

O MATLAB armazena imagens truecolor como uma matriz de dados $m$-by- $n-b y-3$, que define os componentes de cor vermelho, verde e azul (RGB) para cada pixel individual. Imagens truecolor não utilizam um mapa de cores. A cor de cada pixel é determinada pela combinação da intensidade de vermelho, verde e azul armazenados em cada plano de cor na localização do pixel.

Filho e Neto (1999) relatam que o uso de cores em processamento digital de imagens origina-se de fatores motivantes, sendo os principais: na análise automática de imagens (reconhecimento de padrões), onde a cor aparece como um poderoso descritor das propriedades de um objeto, que pode simplificar sua identificação e segmentação; e na análise de imagens com intervenção humana, já que o olho humano pode discernir milhares de nuanças de cores de diferentes tonalidades e intensidades, enquanto sua capacidade de distinguir diferentes tons de cinza não passa de algumas poucas dezenas de tons diferentes.

O processamento digital de imagens é o conjunto de técnicas para a manipulação de imagens digitais com o objetivo de facilitar a extração de informações desejadas. MATLAB é uma linguagem simples e de alto nível de aplicação. Esse programa possibilita a realização de operações matemáticas sobre matrizes, porém tem alcançado uma maior flexibilidade na utilização, ganhando atualmente propósitos mais amplos (BECKER et al., 2010).

\section{MATERIAIS E MÉTODOS}

Antes de serem apresentados os materiais e métodos, o fluxograma da Figura 3 mostra de forma sucinta os processos envolvidos e os produtos gerados, alcançando o objetivo principal: geração de mapa de danos. 


\section{Figura 3 - Fluxograma das etapas envolvidas no trabalho}

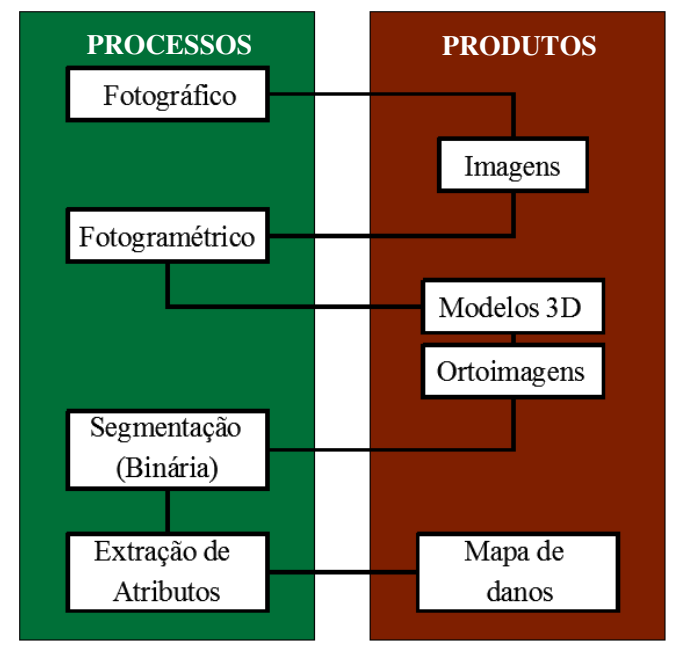

Fonte: Desenvolvida pelos autores

A seguir, serão apresentados os materiais utilizados na pesquisa seguidos dos métodos empregados para a geração dos mapas de danos.

\subsection{Materiais}

Para a aquisição das imagens, foi utilizada a câmera digital Sony RX 100 de alta resolução (20,2 megapixels ou 5472 x 3648 pixels), com sensor equivalente à 13,2 x 8,8 mm, estabelecendo-se os seguintes parâmetros:

- distância focal: $10 \mathrm{~mm}$; e distância focal equivalente a $35 \mathrm{~mm}$ igual a 28 $\mathrm{mm}$

- abertura máxima: f1.8;

- escala de número f: f4.5;

- tempo de exposição: $1 / 1250$ segundos; e

- sensibilidade de ISO: 200.

A câmera foi acoplada a um veículo aéreo não tripulado (VANT), do tipo multirrotor de 4 asas rotativas, desenvolvido no âmbito do projeto microVANT em execução no Departamento de Engenharia Civil e Ambiental da UnB. Esse equipamento possui autonomia de voo de 13 minutos, sistema de controle e estabilização automáticos, motorização elétrica com uso de baterias de lítio, fixação da posição de aquisição das imagens com uso de bússola eletrônica e coordenadas obtidas com GPS. A vantagem do emprego específico do multirrotor foi a possibilidade de voos paralelamente ao plano das fachadas, possibilitando a aquisição de imagens de todas as partes da fachada.

\subsection{Métodos}

\subsubsection{Obtenção de ortoimagem}

Inicialmente, as imagens foram carregadas num programa de fotogrametria digital, o PhotoScan da AgiSoft LLC., com o objetivo de criar um modelo tridimensional da edificação (FIGURA 4 (a)), com as características reais da superfície no momento da capturada da imagem fotográfica. Posteriormente, foi gerada a ortoimagem do plano da fachada a ser mapeado (FIGURA 4 (b)). Ortoimagens são produtos da fotogrametria 
digital e possuem como características a transformação de uma imagem fotográfica (com inclinação do eixo da câmera e mudanças de profundidade) em uma imagem ortográfica, ou seja, eliminam os efeitos da perspectiva.

\section{Figura 4 - Modelo tridimensional da Biblioteca Central da UnB gerado no PhotoScan (a) e ortoimagem de uma das fachadas da Biblioteca Central da $\operatorname{UnB}(\mathbf{b})$}

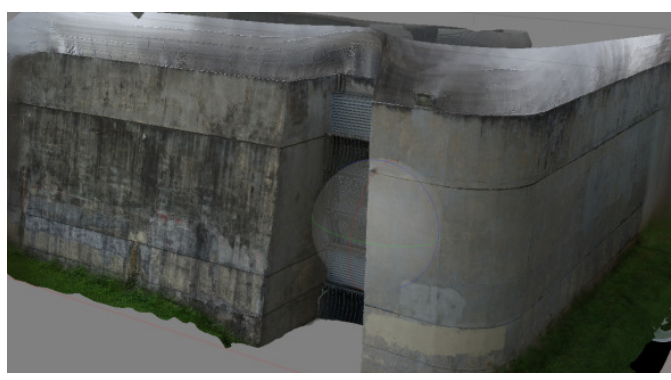

(a)

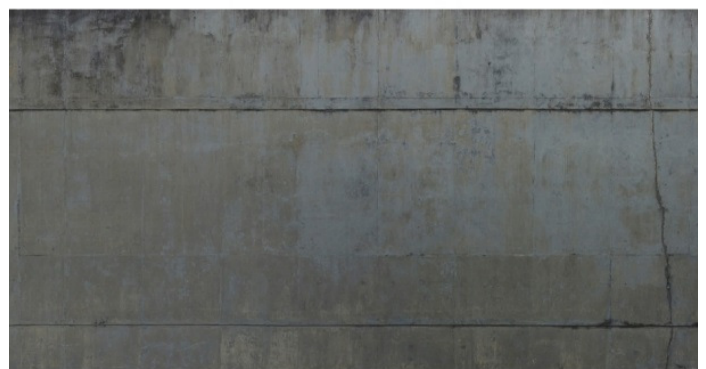

(b)

Fonte: Desenvolvida pelos autores

\subsubsection{Geração de mapas de danos}

Após a etapa de obtenção da ortoimagem, essa foi inserida por comando específico no MATLAB. Para determinação do tamanho da fachada na ortoimagem, foi criado um código para leitura da quantidade de pixels dentro de uma determinada distância conhecida da edificação, conforme Figura 5. A distância correspondeu a 0,98 m e a quantidade de pixels nessa distância foi de 293,46 pixels.

\section{Figura 5 - Verificação da quantidade de pixels em distância conhecida na fachada da Biblioteca Central da UnB}

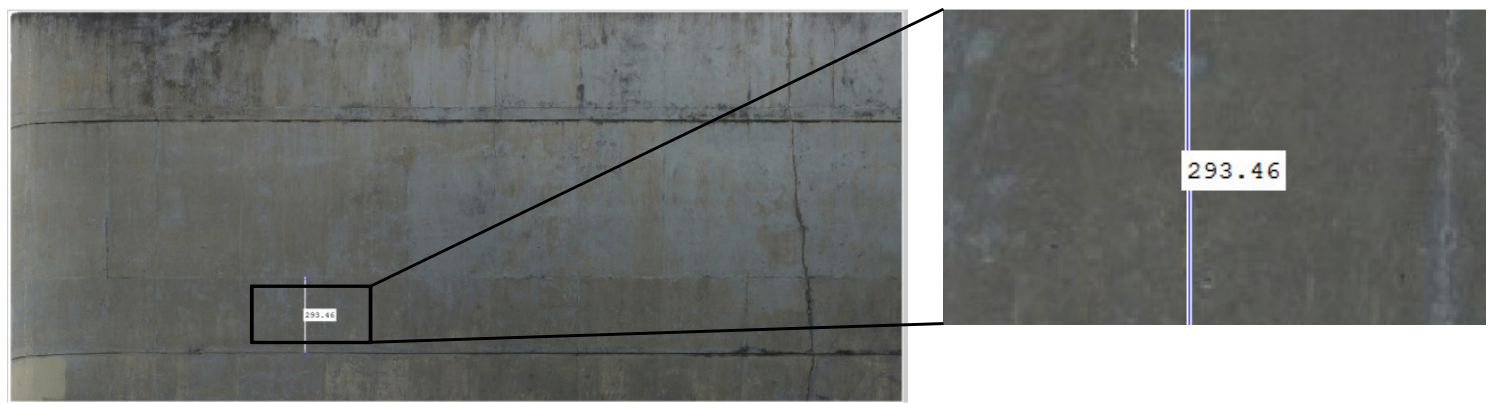

Fonte: Desenvolvida pelos autores

Assim, conhecendo-se o tamanho do pixel pode-se calcular a área da fachada na ortoimagem em função da quantidade de pixels na vertical e na horizontal. Para uma fotografia comum, esse procedimento não seria necessário, pois para fotografias padrão o valor da quantidade de pixels já é conhecido.

Cumprida essa etapa, subdividiu-se a ortoimagem em partes, na qual cada parte corresponde a uma característica computacional, nesse caso, manchas e elementos da fachada. Especificou-se a área mínima das manchas a serem encontradas. Essa etapa é conhecida na área de processamento digital de imagens como segmentação, ilustrada na Figura 6. 
Figura 6 - Segmentação de imagem da Biblioteca Central da UnB

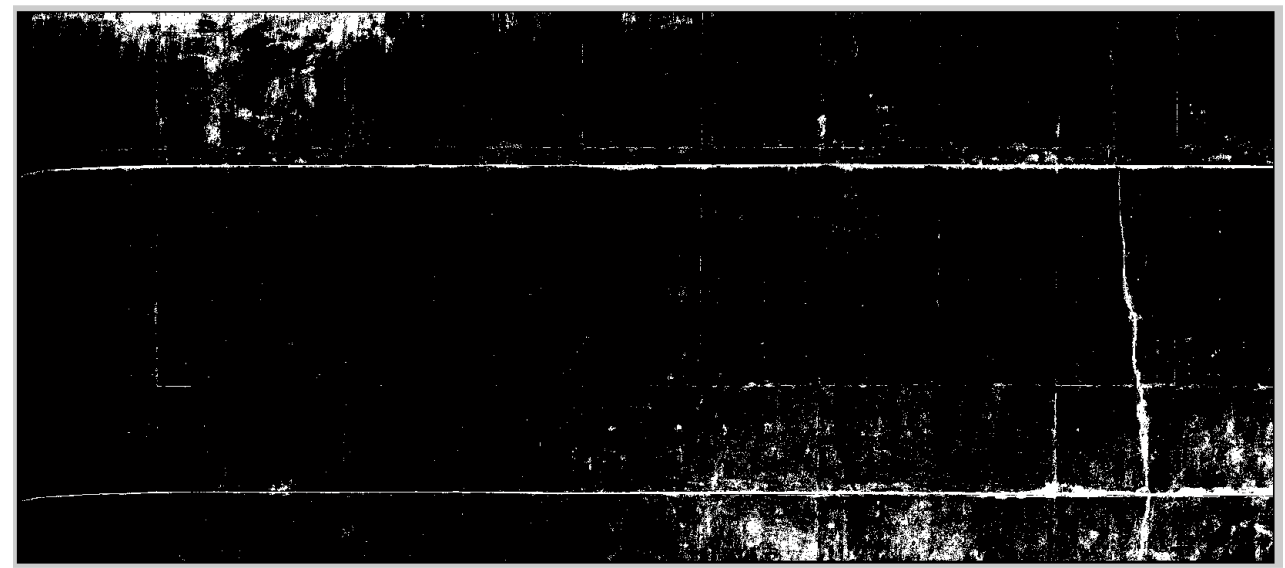

Fonte: Desenvolvida pelos autores

Posteriormente, o código baseado em contorno das características identificadas na segmentação foi criado, onde as áreas de manchas escuras acentuadas foram mapeadas. Após identificação dessa área, foi calculado o percentual e a área da fachada com essas manifestações patológicas.

\section{RESULTADOS E ANÁLISES}

O mapa de danos de uma das fachadas da Biblioteca Central da UnB foi gerado, com resultado satisfatório. Para esse plano de fachada com área de $64,17 \mathrm{~m}^{2}$, encontrou-se uma área de manchas de $1,98 \mathrm{~m}^{2}$ para manchas com áreas maiores que $12 \mathrm{~cm}^{2}$, correspondendo à 3,09\% de manchas escuras acentuadas. Essas manchas foram circundadas por linhas na cor amarela, como ilustrado na Figura 7.

\section{Figura 7 - Mapa de danos da fachada da Biblioteca Central da UnB para as manchas maiores que $12 \mathrm{~cm}^{2}$}

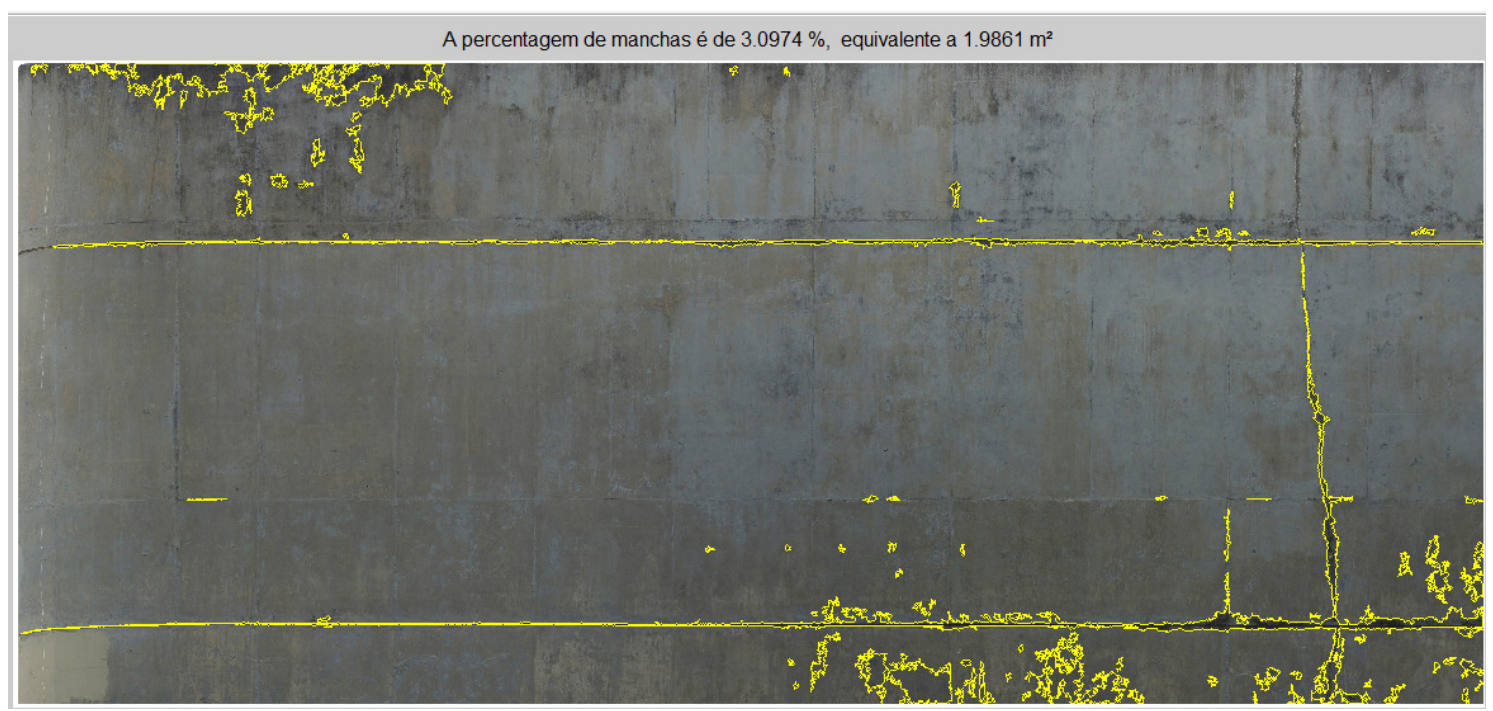

Fonte: Desenvolvida pelos autores

Para um resultado mais criterioso e para a mesma fachada, foi criado um mapa de danos para manchas maiores que $0,6 \mathrm{~cm}^{2}$, resultando num maior percentual de manchas, $3,69 \%$, e uma área de $2,37 \mathrm{~m}^{2}$, como ilustrado na Figura 8. 


\section{Figura 8 - Mapa de danos da fachada da Biblioteca Central da UnB para as manchas maiores que $0,6 \mathrm{~cm}^{2}$}

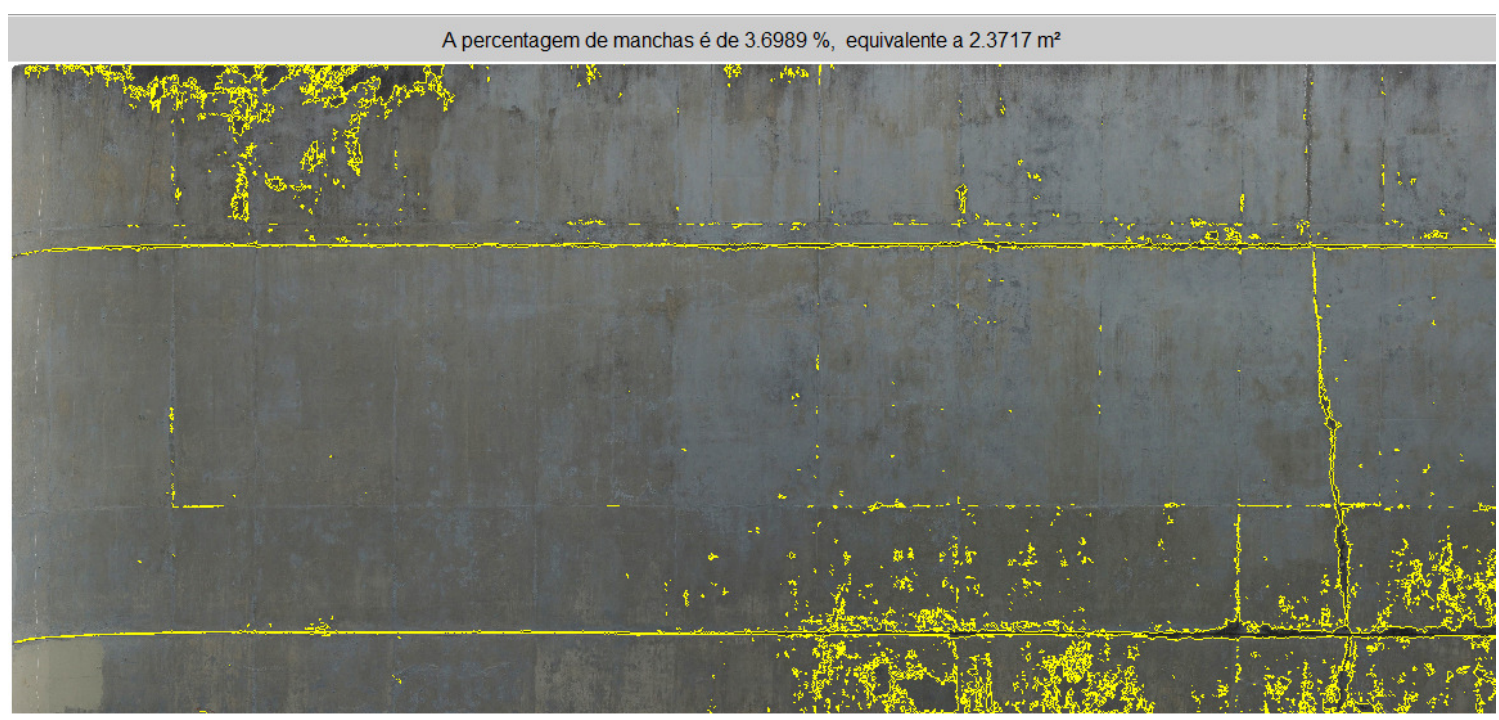

Fonte: Desenvolvida pelos autores

Nota-se que existem outras manchas na fachada que não foram mapeadas, porém buscou-se nesse trabalho por padrão de cor, identificar somente as manchas mais escuras. As demais manchas poderiam ser mapeadas, no entanto o padrão de cor a ser especificado no código seria outro.

\section{CONSIDERAÇÕES FINAIS}

A obtenção de ortoimagens pelo PhotoScan demonstrou-se uma excelente técnica para correção do efeito de perspectiva, gerando as representações da fachada da biblioteca sem qualquer ângulo de inclinação, ou seja, propiciando a representação gráfica ortogonal dessa fachada. O uso de VANT também foi importante para alcançar o topo da edificação e obter imagens mais próximas e melhorar a resolução das fotografias.

A metodologia para geração de mapa de danos de fachadas por extração automática de informações em MATLAB demonstrou-se possível e aplicável às manifestações patológicas por formação de manchas. Outros tipos de manchas, como lixiviação de carbonato de cálcio e oxidação, também são passíveis de reconhecimento por essa técnica.

Conclui-se por meio desse estudo que se torna possível, utilizando o método apresentado no presente trabalho, acompanhar a evolução de manifestações patológicas em função do tempo, visando medidas preditivas de manutenção das fachadas de edificações, assim como de outros tipos de construção.

\section{REFERÊNCIAS}

BARTHEL, C., LINS, M., PESTANA, F. O papel do mapa de danos na conservação do patrimônio arquitetônico. In: CONGRESSO IBEROAMERICANO Y VIII JORNADA TÉCNICAS DE RESTAURACIÓN E CONSERVACIÓN DEL PATRIMÔNIO, 2009. La Plata, Buenos Aires, Argentina. 20 p.

BECKER, A.J., SILVA, D.M.I., DIAS, F.H.S., PINHEIRO, L.K . Noções Básicas de Programação em MATLAB. 2010. 
BERTRAND, E., BOOTH, R., BRANDT, E., DIAZ, M.L., FLUELER, P., HUTCHINSON, T., JAISINGH, L., KALINGER, P., KANE, E., KYLE, B., LELONG, M., PAROLI, R., PUTERMAN, M., ROBERTS, K., ROSSITER, W., SARTORI, P., SAUNDERS, G., SIQUEIRA, F., SMITH, T., TANAKA, K., VANDEWYNCKEL, J. Condition Assessment of Roofs. Joint Committee CIB W83/RILEM TC 166-RMS. CIB Publication 289. Rotterdam: International Council for Research and Innovation in Building and Construction (CIB), 2003, $24 \mathrm{p}$.

CARRIÓ, J.M. Arquitetura, arte funcional. Informes de la Construcción, Madrid, Instituto Eduardo Torroja, v. 37, n.374, p. 5-16, 1985.

COSTA, L.G.G. Cronidas: Elaboração da base de dados para auxílio em representação de mapa de danos, 2010. Dissertação para obtenção do grau de Mestre em Arquitetura e Urbanismo. Universidade Federal da Bahia.

COSTA, L. G. G. Cronidas: Uma proposta de base de dados para confecção de mapas de danos em edificações de interesse histórico-cultural. In: SIGRADI 2011 - XV CONGRESO DE LA SOCIEDAD IBEROAMERICANA DE GRÁFICA DIGITAL, 2011, Santa Fé. Anais... Santa Fé: FADU-UNL, 2011. P. 56-59.

Flores-Colen, I. Metodologia de avaliação do desempenho em serviço de fachadas rebocadas na óptica da manutenção predictiva. Tese de doutoramento, 2009, 487 p.

GASPAR, P. M. dos S. L. Vida útil das construções: Desenvolvimento de uma metodologia para a estimativa da durabilidade de elementos da construção. Aplicação a rebocos de edifícios correntes. 2009. 358f. Tese para a obtenção do grau de Doutor em Ciências de Engenharia. Universidade Técnica de Lisboa. Instituto Superior Técnico.

MARQUES NETO, O. VIEIRA NETO, H. Processamento Digital de Imagens. Rio de Janeiro: Brasport, 1999.

PETRUCCI, H.M.C. A alteração da aparência das fachadas dos edifícios: interação entre as condições ambientais e a forma construída. 2000. Dissertação de Mestrado. Universidade Federal do Rio Grande do Sul.

TINOCO, J.E.L. Mapa de danos: Recomendações básicas. Centro de estudos avançados da conservação integrada - CECI, v. 43, Olinda, Pernambuco, 2009. 